\title{
A 2.3/3.3 GHz Dual Band Antenna Design for WiMax Applications
}

\author{
Adit Kurniawan, Iskandar \& P.H. Mukti \\ School of Electrical Engineering and Informatics, Bandung Institute of Technology, \\ Email: adit@1trgm.ee.itb.ac.id
}

\begin{abstract}
A triangular-slot antenna with rectangular patch for $2.3 / 3.3 \mathrm{GHz}$ WiMax applications has been implemented on DICLAD 527 substrate (relative permittivity $\varepsilon r=2.5$ ) with $1.524 \mathrm{~mm}$ of substrate thickness. A rectangular patch printed on one side of the substrate is fed by a $50 \Omega$ microstrip line and acts as the frequency tuning stub, while the triangular slot is positioned on the opposite side of the substrate, center lined to the rectangular stub. From the measurement results based on VSWR $=2$ or equal to the return loss of $9.53 \mathrm{~dB}$, at the lower band of $2.3 \mathrm{GHz}$ the resulting impedance bandwidth is $290 \mathrm{MHz}$ (from 2.16 to $2.45 \mathrm{GHZ}$ ) and at the upper band of $3.3 \mathrm{GHz}$ is $370 \mathrm{MHz}$ (from 3.31 to $3.68 \mathrm{GHz}$ ), providing services for $2.3 \mathrm{GHz}$ and $3.3 \mathrm{GHz}$ frequency bands allocated for WiMax applications. The antenna gain measurement at $2.3 \mathrm{GHz}$ frequency band is almost agrees with the simulation result of $3.2 \mathrm{dBi}$. While at 3.3 $\mathrm{GHz}$ the gain is approximately $4.4 \mathrm{dBi}$ and continues to decrease with increasing frequency. The antenna gain measurement achieves maximum of 4.8 $\mathrm{dBi}(6 \mathrm{dBi}$ from simulation) at about $3 \mathrm{GHz}$. The simulation and measurement results are evaluated and discussed.
\end{abstract}

Keywords: antenna; dual-band; rectangular-stub; triangular-slot; wimax.

\section{$1 \quad$ Introduction}

Rapid progress in wireless communication services have led to an enormous challenge in antenna design. Benefited from unlicensed bands, WLAN attracts attention of many customers and researchers around the world. Many devices using WLAN to take advantages from high speed connectivity between PCs, laptops, cell phone, and other equipment in corporate, public, and home environments. In the near future, WiMax technology with different standards is going to come to market. Wireless data services have evolved and continue to grow using various technologies, such as $2 \mathrm{G} / 3 \mathrm{G}$ that evolves towards Long Term Evolution (LTE), as well as WiFi and WiMax technology. The impact of such diverse technologies is on the use of frequency band in that different technologies will need to occupy different frequency allocations. WiMax technology, both for nomadic application (frequency band of 2.3-2.4 GHz) and mobile application (frequency band of 3.3-3.7 GHz) is likely to be a prominent candidate to serve for wireless data in the near future. Therefore there is a need 
to develop a dual band antenna for both WiMax applications occupying 2.3/3.3 $\mathrm{GHz}$ frequency bands. There are several papers on dual-band antennas for $802.11 \mathrm{a} / \mathrm{b} / \mathrm{g}$ standards. Liu et. al. [1] proposed inverted-F antenna that can cover the 2.4/5.2 GHz WLAN bands. Raj et al. [2] proposed coplanar antenna printed on FR4 that operates on 2.4/5.2/5.8 GHz bands. Wu et al. [3] reported dual broadband slot antenna, in which two wide resonances were obtained by using a U-shaped strip inset at the center of the slot antenna on a substrate with relative permittivity of 4.7. Another technique to make a compact multi band antenna can be found in [4] and [5], which also describe many issues related to advancement in compact microstrip antenna technologies.

In this paper, we propose a triangular-slot antenna combined with rectangular patch as the tuning stub to produce a dual band antenna operating at 2.3/3.3 $\mathrm{GHz}$ bands that can support both nomadic and mobile WiMax applications. The proposed antenna uses DICLAD material substrate that can provide larger gain compared to that of FR4 material due to lower value of relative permittivity (relative permittivity of DICLAD 527 is 2.5 compared to 4.4 of FR4). However, the use of low cost material FR4 is more attractive, but should be using a more complex array design to compensate for the antenna gain.

This paper is organized as follows. Section 2 presents the antenna design and simulation, Section 3 shows the antenna fabrication and measurement. Analysis and discussion are described in section 4. Finally, Section 5 concludes the paper.

\section{$2 \quad$ Antenna Design and Simulation}

The proposed antenna design in this paper refers to the antenna shape that was produced in [5] using a triangular slot with rectangular tuning stub for 2.4/5.2/5.8 GHz triple-band WLAN applications (IEEE 802.11b/a standard), which is implemented on FR4 substrate materials. In this paper we develop a similar method to design a dual band antenna operating at $2.3 / 3.3 \mathrm{GHz}$ for WiMax nomadic and mobile applications. We also use DICLAD (relative permittivity $\varepsilon_{r}=2.5$, with substrate thickness $h=1.54 \mathrm{~mm}$ ), instead of FR4 (relative permittivity $\varepsilon_{r}=2.5$ ) as in [5] in order to evaluate different antenna characteristics and trade-off for better antenna gain. WiMax technology covers the IEEE 802.16d standard for nomadic users using 2.3-2.4 GHz frequency band and IEEE 802.16e for mobile users using 3.3-3.7 GHz frequency band. Therefore 2.3/3.3 GHz dual band antennas are proposed in this paper for the sake of simple and common antenna usage. The antenna bandwidth and gain specifications are expected to satisfy various applications at different countries. Usually the antenna requires $95 \mathrm{MHz}$ of bandwidth $(\mathrm{SWR}=2)$ at $2.3-2.95 \mathrm{GHz}$ frequency band and wider bandwidth can be obtained in the $3.3-3.7 \mathrm{GHz}$ 
frequency band. The required antenna gain varies with the application and the required area of coverage. For access points onboard the vehicle, e.g. buses or trains, 3-4 $\mathrm{dBi}$ of antenna gain will be acceptable, while for access points of larger areas the required antenna gains of up to ten $\mathrm{dBi}$ are needed.

Figure 1 shows the geometry of the proposed dual-band antenna. The antenna is intended to operate in $2.3 / 3.3 \mathrm{GHz}$ frequency bands as stated by $802.16 / \mathrm{d} / \mathrm{e}$ specification for nomadic and mobile WiMax applications. The lower band covers $2.3 \mathrm{GHz}$ WiMax $802.16 \mathrm{~d}$ standard, and the upper band covers $3.3 \mathrm{GHz}$ WiMax 802.16.e standard. The proposed antenna consists of triangular slot and rectangular tuning stub printed on the opposite side. Antenna occupies an area of $100 \times 92.5 \mathrm{~mm}^{2} . L_{l}$ is the length of each side of the equilateral triangle, while the length and width of the rectangular tuning stub is $W_{l}$ and $W_{2}$, respectively. The length of microstrip feed line from the feed point to the rectangular stub is denoted by $G_{2}$ while $G_{1}$ is the gap between rectangular tuning stub and triangular slot on the opposite side. The rectangular patch as a tuning stub is fed by a $50 \Omega$ microstrip line and is printed on the opposite side of microwave substrate (DICLAD) and placed symmetrically with respect to the centerline of triangular slot. The use of tuning stub has been shown to be effective for the resonant frequency adjustment of microstrip antennas [6]. The dual-band characteristic is obtained from dual resonant structures having different dimensions of the rectangular tuning stub, while the triangular slot affects and modifies the ground plane characteristics of the overall antenna construction.

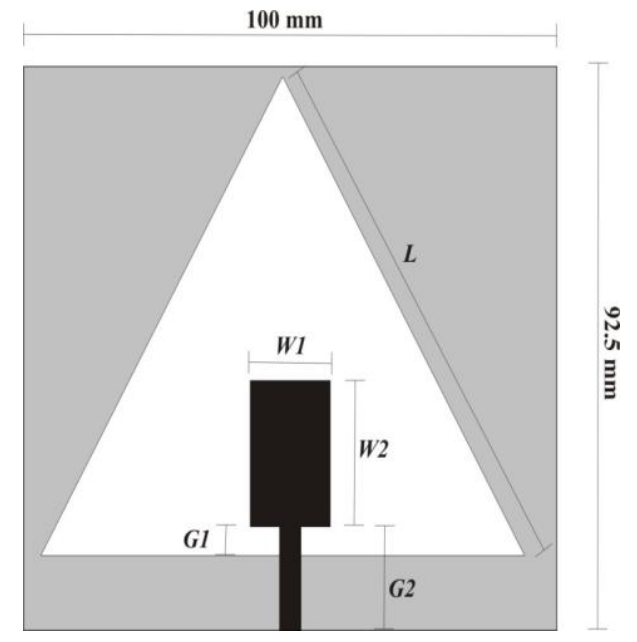

Figure 1 Geometry of the proposed dual-band antenna.

Simulation process involves the selection of materials for substrate, tracing and choosing the ground plane dimension, drawing the models, choosing the 
excitation port, and setting up the optimum geometric dimension to satisfy the desired center frequency, as well as the bandwidth requirement for each band for the specified return loss/VSWR requirements. The simulation is iteratively conducted until the desired results are found. Referring to Figure 1 the optimum dimensions of the proposed geometric antenna are as follows: $L=84.3 \mathrm{~mm}, W_{l}$ $=15.3 \mathrm{~mm}, W_{2}=24.2 \mathrm{~mm}, G_{1}=4,7 \mathrm{~mm}, G_{2}=17 \mathrm{~mm}$ and the width of feed line is $4 \mathrm{~mm}$.

As mentioned in [5] that by choosing suitable parameters, such the antenna design can produce significant increase of bandwidth. Figures $2 \& 3$ show the simulated return loss and VSWR characteristics of the proposed dual-band antenna.

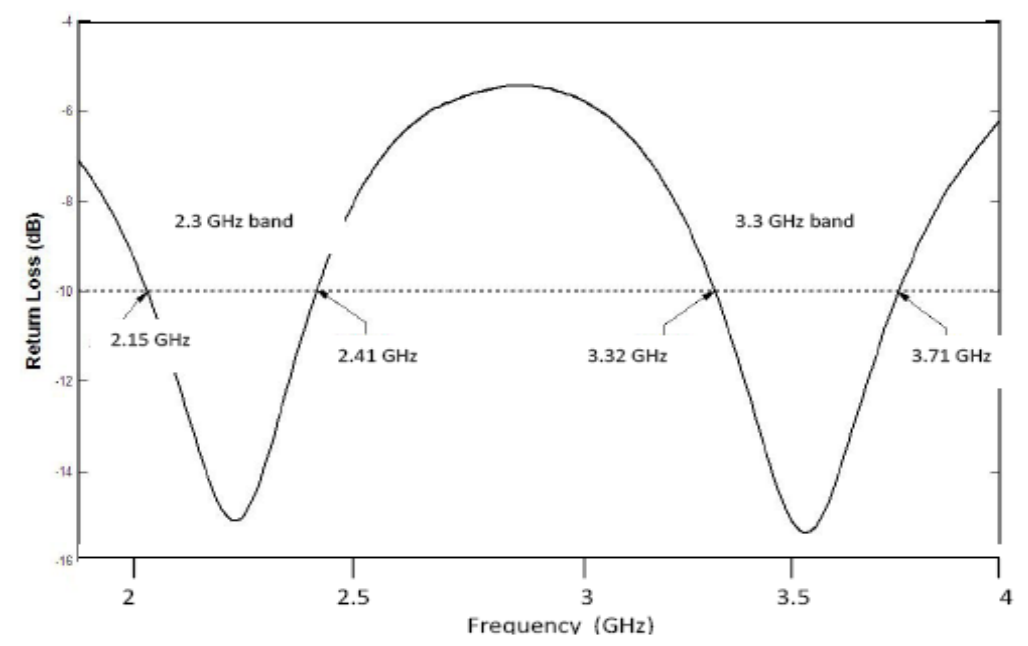

Figure 2 Simulated return loss for proposed dual-band antenna.

From the simulation, the impedance bandwidth of the lower frequency band determined by $-10 \mathrm{~dB}$ return loss shown in Figure 2 (equal to the VSWR of 1.93) reaches approximately $300 \mathrm{MHz}$ of bandwidth $(2.15 \mathrm{GHz}-2.41 \mathrm{GHz})$, which is approximately $13 \%$ of bandwidth for the frequency band of $2.3 \mathrm{GHz}$ ). For the upper frequency band, the impedance bandwidth is wider that reaches approximately $400 \mathrm{MHz}(3.315 \mathrm{GHz}-3.71 \mathrm{GHz}$, about $11 \%$ for the frequency band of $3.3 \mathrm{GHz}$ ). The center frequency, as well as the impedance bandwidth in the two bands are determined by adjusting the rectangular dimensions that needs to be carefully tuned. 


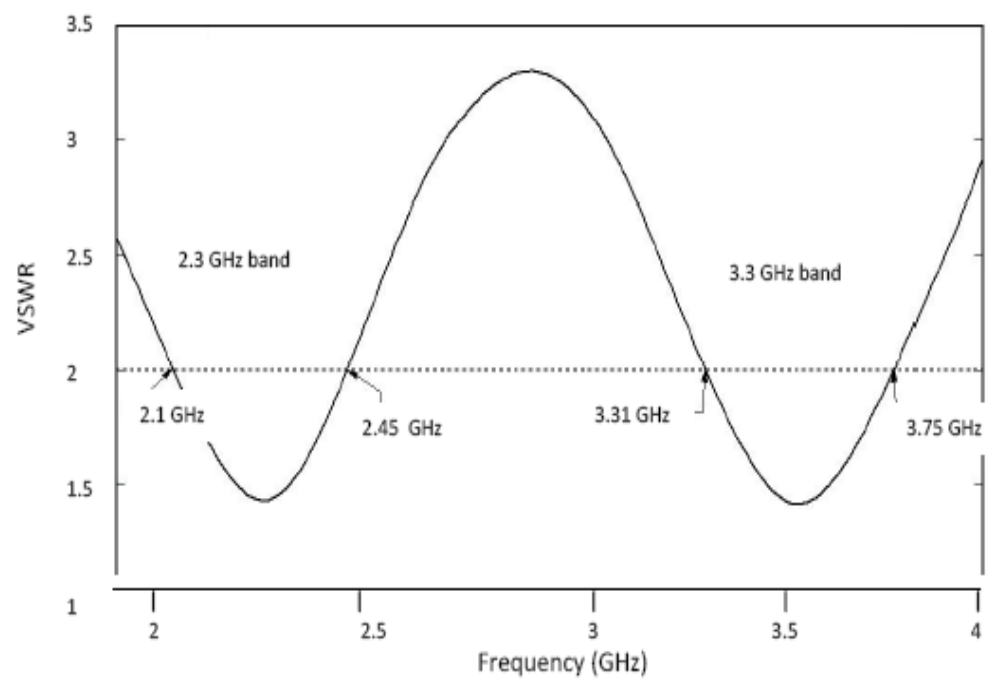

Figure 3 VSWR of the proposed antenna.

With a thorough and extensive simulation, we looked at the effect of the rectangular dimension, as well as the effect of feed length and feed gap on the impedance bandwidth. Note that the feed length $G_{2}$ will determine the gap between the rectangular patch and the triangular slot on the reverse side, $G_{l}$. We found that the impedance bandwidth at $2.3 \mathrm{GHz}$ is quite independent of the patch dimension but is slightly sensitive to the changes of the feed length and/or feed gap. On the other hands, the impedance bandwidth at $3.3 \mathrm{GHz}$ band is sensitive to the changes of the patch dimension but rather independent of the feed length or feed gap. Figures 4 (a)-(c) show the effect of feed length/gap and the patch dimensions on impedance bandwidth and center frequency.

We can clearly see from Figure 4 (a) that the feed length $G_{2}$, which in turn, affects the feed gap, $G_{1}$ changes the impedance bandwidth at both 2.3 and 3.3 $\mathrm{GHz}$ bands and also slightly changes the resonant frequency at $2.3 \mathrm{GHz}$ but doesn't affect the $3.3 \mathrm{GHz}$ band so much. While the patch dimensions $W_{l}$ and $W_{2}$ affect the impedance bandwidth and resonant frequency of the $3.3 \mathrm{GHz}$ (upper) band but not much affect the $2.3 \mathrm{GHz}$ (lower) band. This can be explained that good impedance matching can be obtained by tuning the best coupling between the rectangular patch and the triangular slot, which is performed by adjusting the feed gap $G_{1}$ and, in turn, the feed length $G_{2}$. Therefore the dimension of rectangular patch determines the resonant frequency and impedance bandwidth of the upper $3.3 \mathrm{GHz}$ band, and the dimension of feed gap as well as the feed length tunes the impedance bandwidth and resonant frequency of the lower $2.3 \mathrm{GHz}$ band. 


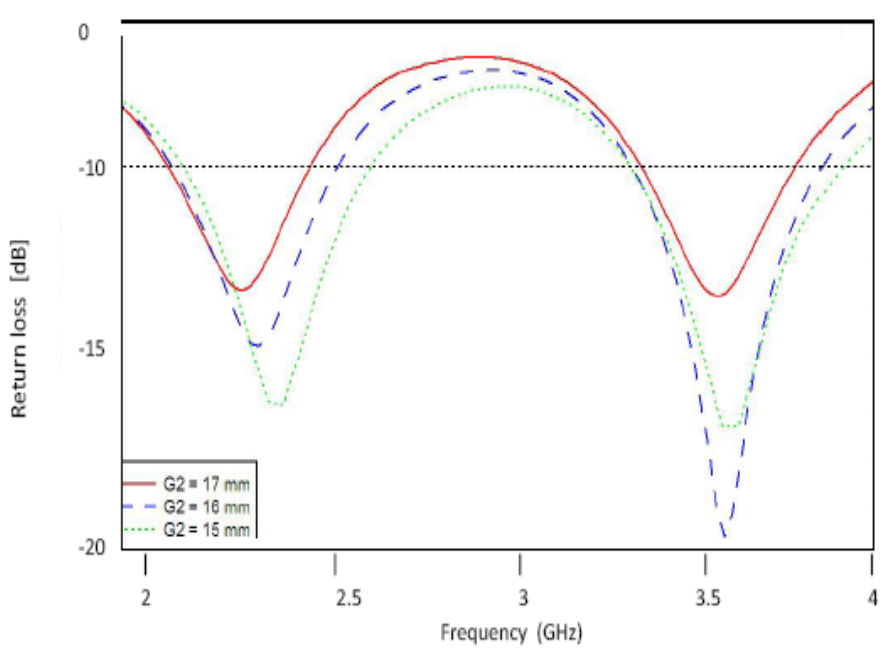

(a)

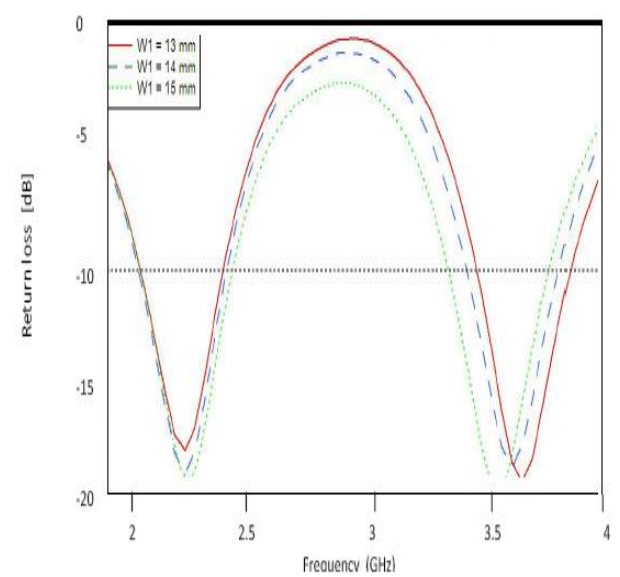

(b)

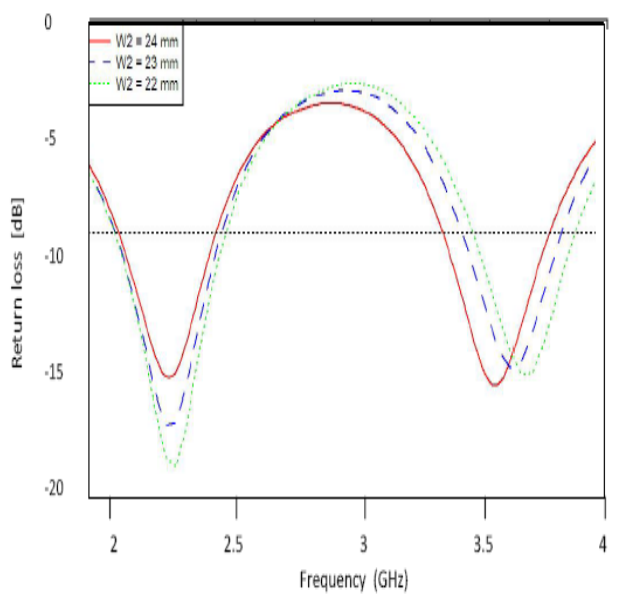

(c)

Figure 4 Effect of feed gap (a), patch dimension $W_{1}$ (b) and $W_{2}$ (c) on center frequency and impedance bandwidth.

Figure 5 shows the antenna gain versus its frequency. From Figure 5, the simulated gain at $2.3 \mathrm{GHz}$ frequency band (after interpolation) is approximately $3.2 \mathrm{dBi}$, and the gain at $3.3 \mathrm{GHz}$ frequency band is approximately $4.4 \mathrm{dBi}$. It can also be seen that the antenna gain achieves maximum level of $4.8 \mathrm{dBi}$ (from measurement) or $6 \mathrm{dBi}$ (from simulation) at approximately $3 \mathrm{GHz}$ and then continue to decline for higher frequencies. 


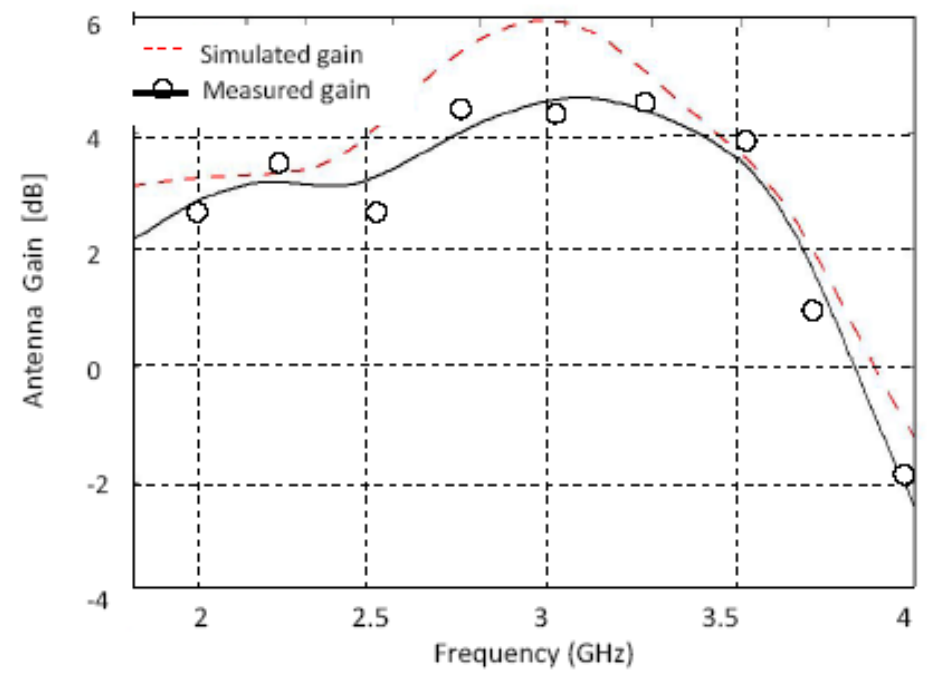

Figure 5 Simulated and measured gain $(\mathrm{dBi})$ of the proposed antenna.

The radiation pattern for azimuth direction shows a bidirectional pattern for both frequency bands. The pattern for elevation direction shows a regular broadside, which is suitable for either access point or mobile terminal application in wireless communication. Pattern in azimuth direction is also suitable for access point with bidirectional coverage application. The simulation results for the radiation pattern are shown in Figure 6.

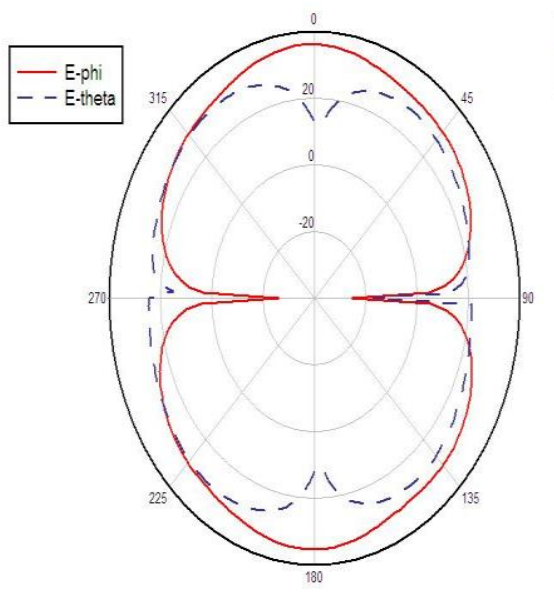

(a) For $2.3 \mathrm{GHz}$ band

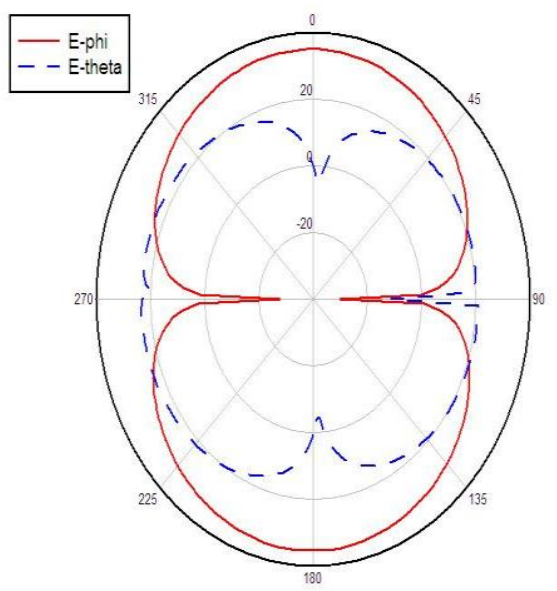

(b) For $3.3 \mathrm{GHz}$ band

Figure 6 Radiation pattern of the proposed antenna. 
We can see from Figure 6 that radiation pattern for both frequency bands are quite similar and comparable, with the pattern of azimuth (phi) direction is more directive for the upper band of $3.3 \mathrm{GHz}$. This corresponds to the fact that the antenna gain is higher for the upper band. The radiation pattern in Figure 6a and $\mathrm{b}$ shows that the pattern for elevation (theta) dropped at 0 degree. This can be explained that radiation is maximum towards the direction that is perpendicular to the radiator plane and minimum towards the direction parallel to the antenna plane of rectangular stub as the radiator. Since theta $=0$ or 180 degrees corresponds to the direction parallel to the antenna plane, its radiation pattern will drop (minimum). Similar behavior occurs on the azimuth plane, so that the radiation pattern will be maximum towards phi $=0$ or 180 degrees and minimum towards phi $=90$ or 270 degrees.

\section{Antenna Fabrication and Measurement}

The proposed antenna is implemented using DICLAD 527 material in order to obtain a higher antenna gain. The use of low cost FR4 material will result in a lower antenna gain, so that to produce a high gain antenna using low cost FR4 materials will require a multi element array design. Figure 7 shows the implemented antenna for single element design.

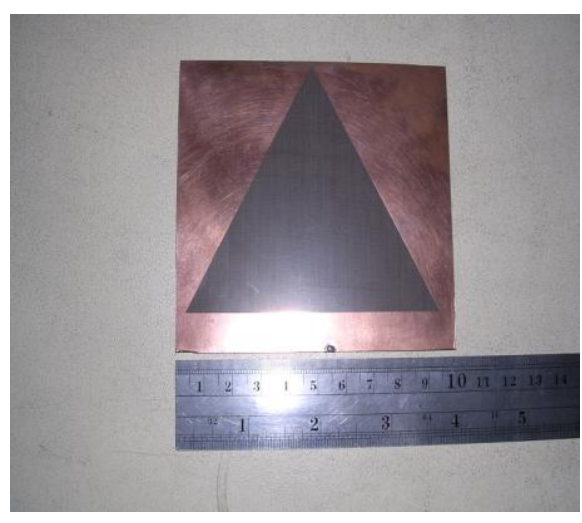

(a) View of Triangular slot

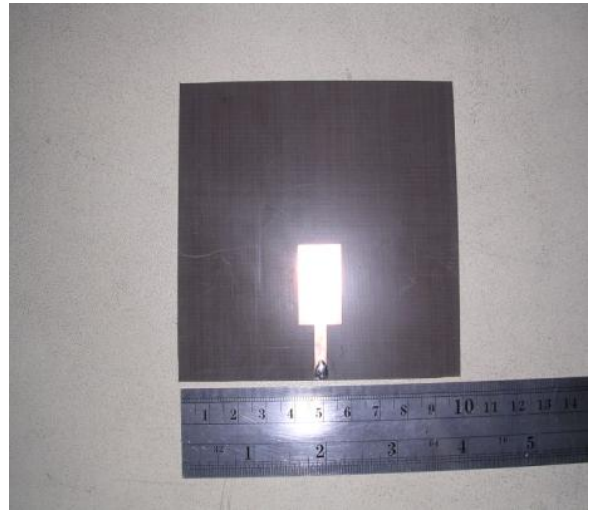

(b) View of rectangular patch

Figure 7 Prototype of the proposed 2.3/3.3 GHz dual band antenna.

A prototype of antenna is fabricated as shown in Figure 7 and tested by measuring its parameters, particularly VSWR and antenna gain, to validate the simulation result as well as to verify the antenna design specification. Measurement of VSWR or return loss is most important because our main intention in this research is to produce a dual band characteristic within the 
specified center frequency with sufficient impedance bandwidth requirement. Here we show the measurement of VSWR and not the return loss, since it can represent similar behavior of the frequency response for the antenna but the bandwidth specification is published based on VSWR. For ease of comparison, Figure 8 shows the measured as well as the simulated VSWR for $2.3 / 3.3 \mathrm{GHz}$ frequency bands.

We can see from Figure 8 that for VSWR $=2$ (equal to return loss of $-9.53 \mathrm{~dB}$ ), at the lower band of $2.3 \mathrm{GHz}$ results in impedance bandwidth of nearly 290 $\mathrm{MHz}$ (from 2.16 to $2.45 \mathrm{GHZ}$ ) and at the upper band of $3.3 \mathrm{GHz}$ results in the impedance bandwidth of $370 \mathrm{MHz}$ (from 3.31 to $3.68 \mathrm{GHz}$ ). When compared with the simulation, the measurement results exhibit close agreement, except for the lower band the bandwidth measurement is $60 \mathrm{MHz}$ lower than the simulation. This impedance bandwidth can meet the specification for WiMax nomadic (2.3-2.4 GHz) as well as for WiMax mobile standard (3.3 to $3.7 \mathrm{GHz})$.

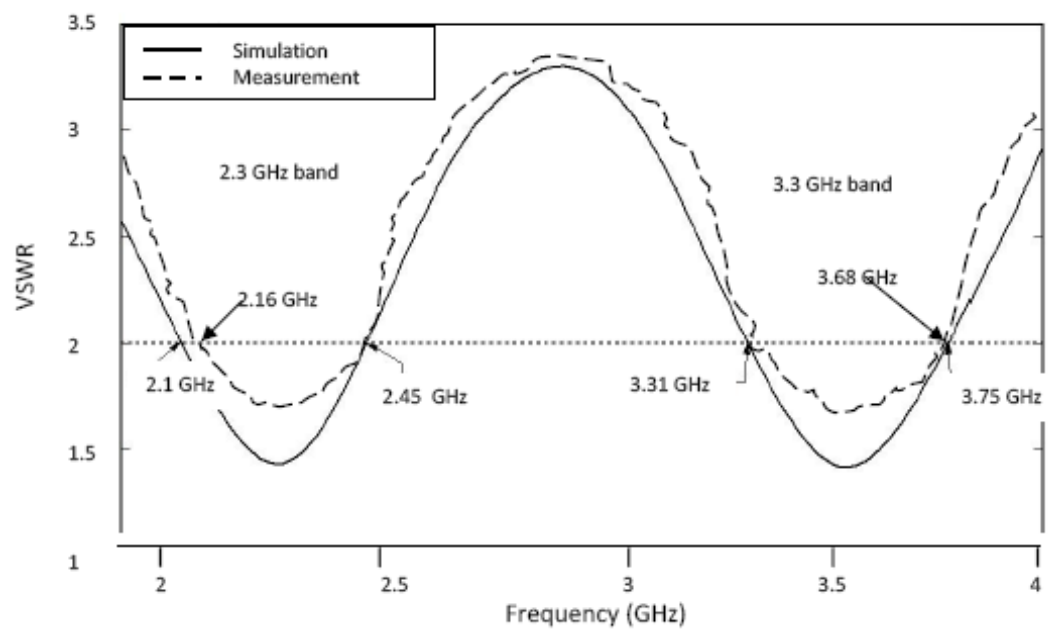

Figure 8 Simulated and Measured VSWR of the proposed antenna.

The gain measurement and simulation presented in Figure 5 shows that the gain obtained from measurement (interpolation of the measurement data) at $2.3 \mathrm{GHz}$ frequency band is approximately $3 \mathrm{dBi}$, which almost agrees with the simulated gain. While the measured gain at $3.3 \mathrm{GHz}$ frequency band is approximately 3.8 $\mathrm{dBi}$, which is $1.4 \mathrm{~dB}$ lower than the simulated gain of $5.2 \mathrm{dBi}$. It can also be seen that the measured antenna gain increases and achieves maximum level of approximately $4.8 \mathrm{dBi}(6 \mathrm{dBi}$ from simulation) at $3 \mathrm{GHz}$ and then continue to decline for higher frequencies. The antenna gain at the upper band $(3.3 \mathrm{GHz}$ band) is higher than that of the lower band $(2.4 \mathrm{GHz})$ due to the fact that although in general the antenna gain reduces with increasing frequency, but in 
this paper, the rectangular patch plays an important role in producing the upper band of $3.3 \mathrm{GHz}$, so that the gain is higher and this fact will be argued in the following section.

\section{Analysis and Discussion}

From extensive simulations we have found the effect of rectangular dimension, as well as the effect of feed length on resonant frequency and the impedance bandwidth. Note that the feed length $G_{2}$ will determine the feed gap between rectangular patch and the triangular slot $G_{l}$. We found that the impedance bandwidth at $2.3 \mathrm{GHz}$ is quite independent of the patch dimension but is slightly sensitive to the changes of the feed length. On the other hands, the impedance bandwidth at $3.3 \mathrm{GHz}$ band is sensitive to the changes of patch dimension but rather independent of the feed length. When the feed line length $G_{2}$ is reduced the resulting center frequency of the lower band $(2.3 \mathrm{GHz})$ slightly shift to the higher frequency band, while the higher frequency band remains stable. The impedance bandwidth also increases when $G_{2}$ value decreases. This aspect can be inferred that to tune on one of the frequency bands, we can vary the gap $G_{2}$. However it needs to be traded off with the impact on the resulting bandwidth.

We also studied the effect of the rectangular stub width $W_{l}$ and its length $W_{2}$ on the resonant frequency and impedance bandwidth. When $W_{l}$ is reduced, while all others parameters are constant, it shows that the upper resonant frequency band increases. However the impact on impedance bandwidth is that it decreases the bandwidth of the lower band but keeps the bandwidth of the upper band unchanged. When the stub height $W_{2}$ is reduced while other antenna dimension is kept constant, the lower resonant frequency decreases, but resulting in the increased impedance bandwidth. At the upper band, decreasing $W_{2}$ results in the shift of the center frequency to a higher frequency, without any significant change of impedance bandwidth. Those behavior can be explained that good impedance matching can be obtained by tuning the best coupling between the rectangular patch and the triangular slot, which is performed by adjusting the gap $G_{1}$ and, in turn, the feed length $G_{2}$. Therefore, in order to obtain the optimum design, simulation need to be carried out extensively with iteration in order to optimize the antenna specification under conflicting parameter settings.

The antenna gain at $2.3 \mathrm{GHz}$ frequency band is approximately $3 \mathrm{dBi}$, and the gain at $3.3 \mathrm{GHz}$ frequency band is approximately $4.4 \mathrm{dBi}$ (simulation shows 5.2 $\mathrm{dBi}$ ). It can also be seen that the antenna achieves maximum level of $4.8 \mathrm{dBi}(6$ $\mathrm{dBi}$ from simulation) at approximately $3 \mathrm{GHz}$ and then continue to decline for higher frequencies. The antenna gain at the upper band is higher than that of the 
lower band. It also shows that the antenna gain exhibits a larger deviation between the simulation and the measurement result compared to that of the lower band. These can be explained due to the fact that the antenna gain is optimized by the resonant frequency, but in this paper the rectangular patch and triangular slot interact one with another in producing the lower and the upper band of $2.3 / 3.3 \mathrm{GHz}$, so that the gain is maximum somewhere between the two bands, despite the fact that the rectangular patch acts as the radiator mainly for generating the $3.3 \mathrm{GHz}$ band. Therefore, the gain for $3.3 \mathrm{GHz}$ band is higher than that for $2.3 \mathrm{GHz}$ band. However the deviation from the simulation becomes larger at the upper band is most likely due to the fact that at the upper band, geometrical dimension might be less accurate because of smaller dimension compared to that of the larger triangular slot, resulting in a higher percentage of dimensional error.

\section{Conclusions}

A dual-band 2.3/3.3 GHz antenna with rectangular tuning stub and triangular slot has been designed and implemented using DICLAD 527 substrate material. The dual band characteristics can completely cover the frequency requirements stated in IEEE 802.16 d/e standards, which cover $2.3-2.4 \mathrm{GHz}$ and $3.3-3.7 \mathrm{GHz}$ frequency bands. Dual-band performance was achieved by choosing optimum parameters for triangular slot as well as for the optimum dimension of the rectangular tuning stub. Extensive experiment is conducted to see the effect of antenna dimension on dual band characteristics to obtain the desired performance. Return loss and/or VSWR were simulated and measurement was carried out to obtain sufficient impedance bandwidth on the desired frequency band. The results show that based on the VSWR $=2$ (equal to return loss of $9.53 \mathrm{~dB}$ ), at the lower band of $2.3 \mathrm{GHz}$ results in impedance bandwidth of nearly $290 \mathrm{MHz}$ (from 2.16 to $2.45 \mathrm{GHZ}$ ) and at the upper band of $3.3 \mathrm{GHz}$ results in the impedance bandwidth of $370 \mathrm{MHz}$ (from 3.31 to $3.68 \mathrm{GHz}$ ). When compared with the simulation, the measurement results exhibit a good agreement, except for the lower band that shows the bandwidth measurement is $60 \mathrm{MHz}$ lower than the simulation. From our experiment it is found that the impedance matching at $2.3 \mathrm{GHz}$ is quite independent of the patch dimension but is sensitive to the changes of the feed gap between rectangular patch and the triangular slot. On the other hands, impedance matching at $3.3 \mathrm{GHz}$ band is sensitive to the changes of patch dimension and the feed gap. This is because good impedance matching can be obtained by tuning the best coupling between the rectangular patch and the triangular slot, which is performed by adjusting the gap between rectangular stub with the triangular slot as well as the feed length of the feed line. The rectangular patch is therefore responsible to produce the dual band of 2.3/3.3.GHz, while the gap and the length of the feed line in conjunction with the rectangular slot and the rest of the conducting material 
forming the ground plane affect the impedance bandwidth of the antenna. The gain measurement at $2.3 \mathrm{GHz}$ frequency band (using interpolation curve) almost agrees with the simulation result of $3.2 \mathrm{dBi}$. While at $3.3 \mathrm{GHz}$ the gain is higher that can achieve $4.4 \mathrm{dBi}$ and continues to decrease with increasing frequency. The maximum gain obtained from measurement achieves $4.8 \mathrm{dBi}(6 \mathrm{dBi}$ from simulation) at about $3 \mathrm{GHz}$. The obtainable gain characteristic from the proposed antenna design is sufficient for WiMax application to serve as access points of small to medium coverage areas. For wider area applications, a multi element array design will be required, and we leave that for our further study.

\section{Acknowledgement}

This research is funded by ITB Research Grant 2010, contract No.: 135/K01.16/PL/2010 and by National Strategic Research Grant DP2M Dikti, 2010. Therefore the authors thank for their supports of financial contributions to this research.

\section{References}

[1] Liu, D. \& Gaucher, B., A branched inverted-F antenna for dual band WLAN applications, Proceedings of the IEEE Antennas and Propagation Society International Symposium, 3, pp. 2623-2626, Monterey, CA, June 2004.

[2] Raj, R.K., Joseph, M., Aanandan, C.K., Vasudevan, K. \& Mohanan, P., A new compact microstrip-fed dual-band coplanar antenna for WLAN applications, IEEE Trans. Antennas Propag., 54(12), 3755-3762, Dec. 2006.

[3] Wu, J.W., Hsiao, H.M., Lu, J.H. \& Chang, S.H., Dual broadband design of rectangular slot antenna for 2.4 and $5 \mathrm{GHz}$ wireless, IEE Electron. Lett, 40(23), Nov. 2004.

[4] Wong, K.L.,Compact and Broadband Microstrip Antennas, New York: John Wiley and Sons, 2002.

[5] Yoon, J., Jeong, G.T. \& Kwak, K.S., Fabrication and measurement of triangular-slot antenna for triple-band $(2.4 / 5.2 / 5.8 \mathrm{GHz})$ antenna with rectangular tuning stub, Microwave and Opt. Technol. Lett., 49(8), Aug. 2007.

[6] Plessis, M.D. \& Cloete, J., Tuning stubs for microstrip patch antennas, IEEE Antennas Propag. Mag, 36, 52-56, Dec.1994. 